\title{
Mitigating artifacts via half-time reconstruction in thermoacoustic tomography
}

Mark A. Anastasio, Jin Zhang, Xiaochuan Pan, Geng Ku, Lihong V. Wang

Mark A. Anastasio, Jin Zhang, Xiaochuan Pan, Geng Ku, Lihong V. Wang, "Mitigating artifacts via half-time reconstruction in thermoacoustic tomography," Proc. SPIE 5697, Photons Plus Ultrasound: Imaging and Sensing 2005: The Sixth Conference on Biomedical Thermoacoustics, Optoacoustics, and Acousto-optics, (25 April 2005); doi: 10.1117/12.589190

SPIE. Event: SPIE BiOS, 2005, San Jose, CA, United States 


\title{
Mitigating artifacts via half-time reconstruction in thermoacoustic tomography
}

\author{
Mark A. Anastasio ${ }^{a}$, Jin Zhang ${ }^{a}$, Xiaochuan $\operatorname{Pan}^{b}$, Geng $\mathrm{Ku}^{c}$ and Lihong V. Wang ${ }^{c}$ \\ ${ }^{a}$ Department of Biomedical Engineering, Illinois Institute of Technology, Chicago, IL 60616 \\ ${ }^{b}$ Department of Radiology, The University of Chicago, Chicago, IL 60637 \\ ${ }^{c}$ Department of Biomedical Engineering, Texas A\&M University, College Station, TX 77843
}

\begin{abstract}
Thermoacoustic tomography (TAT) is an ultrasound-mediated biophotonic imaging modality with great potential for a wide range of biomedical imaging applications. In this work, we demonstrate that half-time reconstruction approaches for TAT can mitigate image artifacts due to heterogeneous acoustic properties of an object. We also discuss how half-time reconstruction approaches permit explicit control of statistically complementary information in the measurement data, which can facilitate the reduction of image variances.
\end{abstract}

Keywords: Thermoacoustic tomography, photoacoustic tomography, generalized Radon transform, image reconstruction

\section{INTRODUCTION}

Thermoacoustic tomography (TAT), also known as optoacoustic or photoacoustic tomography, is an emerging ultrasound-mediated biophotonic imaging modality that has great potential for a wide range of biomedical imaging applications. The hybrid nature of TAT permits it to have the spatial resolution of an ultrasound technique, but the imaging contrast and sensitivity to biological tissue properties of an optical technique. Accordingly, TAT can provide detailed anatomical and functional information that is not produced by conventional imaging techniques. In recent years, exciting experimental studies have demonstrated the great promise of TAT for important applications including cancer detection ${ }^{1-4}$ and neuroimaging. ${ }^{5,6}$

Exact and explicit inversion formulae have been derived for two-dimensional (2D) and three-dimensional (3D) TAT. 4,7 A common feature of these inversion formulae is that they require complete knowledge of the data function acquired on a measurement aperture that encloses the object. We refer to such a data function as a complete data function. The available explicit reconstruction formulae are not applicable when the complete data function is unknown, and will generally result in severe image artifacts when applied inappropriately in such cases. This is problematic for biomedical imaging applications such as breast imaging, ${ }^{1}$ in which it is not feasible to measure a complete data function.

Even if it is possible to acquire, in some situations it may be advantageous to disregard certain temporal components of the complete data function when reconstructing the image. We have demonstrated mathematically $^{8}$ that knowledge of a half-time data function is sufficient for exact image reconstruction in TAT. A half-time data function is one that describes measurements that are truncated in such a way that only the temporal components corresponding to $t<t_{c}$ are recorded at each transducer location. The quantity $t_{c}$ is the time it takes for an ultrasound pulse to travel from the center of the scanning aperture to the detection location. Although analytic reconstruction formulae have not been identified, we have demonstrated that accurate images can be reconstructed from half-time data functions by use of iterative reconstruction algorithms. ${ }^{8,9}$

In this work, we review and investigate numerically the half-time reconstruction problem for TAT. We demonstrate that half-time reconstruction approaches can mitigate image artifacts due to heterogeneous acoustic properties of an object. We also discuss how half-time reconstruction approaches can permit explicit control of statistically complementary information in the measurement data, which can facilitate the reduction of image variances. Computer-simulation studies are conducted to corroborate our theoretical assertions.

Further author information: Send correspondence to Mark A. Anastasio, Ph.D.: E-mail: anastasio@iit.edu 


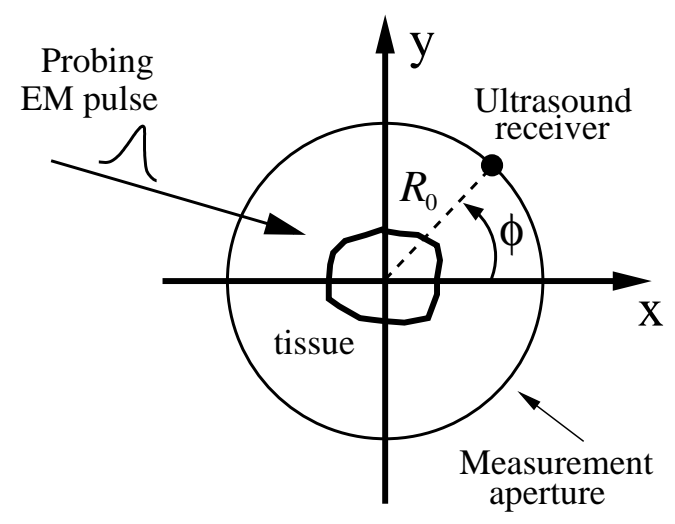

Figure 1. The measurement geometry of TAT. A short electromagnetic pulse irradiates a biological object. The resulting photoacoustic signals are detected by use of wideband unfocused ultrasound transducers that span a measurement aperture.

\section{REVIEW OF THERMOACOUSTIC TOMOGRAPHY}

\subsection{Basic principles of photoacoustic tomography}

In TAT, an electromagnetic pulse (e.g., laser or microwave) is used to irradiate biological tissue. When the electromagnetic pulse is absorbed by the tissue, a photoacoustic effect results in the generation of a pressure wavefield $p(\vec{r}, t)$. After propagating through the object, these wavefields can be measured by use of wideband unfocused ultrasonic transducers that reside on a measurement aperture. A schematic of a two-dimensional (2D) TAT measurement geometry is shown in Fig. 1. In this case, the measurement aperture $\Omega_{0}$ is a circle of radius $R_{0}$ that surrounds the compactly supported object. For the $3 \mathrm{D}$ problem, we will take the measurement to be a sphere of radius $R_{0}$ that enclosed the object. The objective of TAT is to reconstruct an image that represents a map of the spatially variant electromagnetic absorption properties of the tissue.

\subsection{Conventional imaging model}

Assuming that the tissue has homogeneous acoustic properties (but spatially varying electromagnetic absorption properties), the pressure wavefield $p\left(\vec{r}_{0}, t\right)$ that is measured as a function of time at location $\vec{r}_{0} \in \Omega_{0}$ can be expressed as

$$
p\left(\vec{r}_{0}, t\right)=\eta \int d \vec{r} A(\vec{r}) \frac{d}{d t} \frac{\delta\left(t-\frac{\left|\vec{r}_{0}-\vec{r}\right|}{c}\right)}{4 \pi\left|\vec{r}_{0}-\vec{r}\right|},
$$

where $A(\vec{r})$ denotes the energy absorption function, $c$ is the constant speed of sound in the object and homogeneous background medium, and $\eta$ represents the isobaric volume expansion coefficient divided by the specific heat of the object. The image reconstruction problem of TAT is to determine $A(\vec{r})$ from complete knowledge of the data function $p\left(\vec{r}_{0}, t\right)$ by inversion of Eqn. (1).

A mathematical relationship between $p\left(\vec{r}_{0}, t\right)$ and spherical Radon transform can be derived readily. ${ }^{10}$ Specifically, by acting $\int_{0}^{t_{0}} d t$ on both sides of Eqn. (1), one can show that

$$
g\left(\vec{r}_{0}, \bar{t}\right)=\int d^{3} \vec{r} A(\vec{r}) \delta\left(\bar{t}-\left|\vec{r}_{0}-\vec{r}\right|\right)
$$

where

$$
g\left(\vec{r}_{0}, \bar{t} \equiv c t_{0}\right) \equiv \frac{4 \pi}{\eta} t_{0} \int_{0}^{t_{0}} d t p\left(\vec{r}_{0}, t\right)
$$

corresponds to the spherical Radon transform of $A(\vec{r})$. Consequently, image reconstruction in TAT can be accomplished by inversion of Eqn. (2) by use of a reconstruction algorithm for reflectivity tomography. ${ }^{11,12}$ 


\subsection{Half-time image reconstruction paradigm}

Consider the half-time data function $g^{h t}\left(\vec{r}_{0}, \bar{t}\right)$ that is defined as

$$
g^{h t}\left(\vec{r}_{0}, \bar{t}\right)=\left\{\begin{array}{lll}
g\left(\vec{r}_{0}, \bar{t}\right) & : & 0 \leq \bar{t} \leq R_{0} \\
0 & : & \text { otherwise }
\end{array}\right.
$$

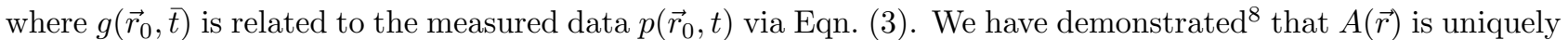
determined by knowledge of $g^{h t}\left(\vec{r}_{0}, \bar{t}\right)$. An identical conclusion was derived by Finch et. al. ${ }^{12}$ This reflects that a two-fold redundancy exists in the complete data function $g\left(\vec{r}_{0}, t\right)$.

Analytic inversion formulae for reconstructing images from half-time data functions have not been identified. However, because Eqn. (2) is a positive integral equation, the expectation maximization (EM) algorithm ${ }^{13}$ can be employed for reconstruction of $A(\vec{r})$. The EM algorithm is easy to implement numerically, and has been utilized in our previous studies of reflectivity tomography. ${ }^{14}$

\section{MOTIVATION FOR HALF-TIME IMAGE RECONSTRUCTION}

Below we reveal that half-time reconstruction approaches can mitigate image artifacts due to heterogeneous acoustic properties of an object. We also show that they permit explicit control of statistically complementary information that can result in the optimal reduction of reconstructed image variances.

\subsection{Mitigation of image artifacts from acoustic heterogeneities}

It is customary in TAT to assume that the object's acoustic properties are homogeneous, and equal to those of the uniform background. However, in many biomedical applications of TAT this assumption may not be reasonably valid. ${ }^{15}$ In these cases there will be inconsistencies between the true and assumed forms of the measurement data, and images reconstructed by inverting Eqn. (2) can contain significant distortions and artifacts. The effects of acoustic heterogeneities in TAT have been studied by $\mathrm{Xu}$ and Wang. ${ }^{15}$ For breast imaging applications, they demonstrated that amplitude distortions were negligible as compared to phase aberrations of the detected pressure wavefields.

With consideration of phase aberrations induced by acoustic heterogeneities, the TAT imaging model can be expressed as

$$
\hat{p}\left(\vec{r}_{0}, t\right)=\eta \int d \vec{r} A(\vec{r}) \frac{d}{d t} \frac{\delta\left[t-t_{f}\left(\vec{r}, \vec{r}_{0}\right)\right]}{4 \pi\left|\vec{r}_{0}-\vec{r}\right|},
$$

where $t_{f}\left(\vec{r}, \vec{r}_{0}\right)$ is the time-of-flight (TOF) of a pressure wave traveling from point $\vec{r}$ to $\vec{r}_{0}$. When the magnitudes of the acoustic heterogeneities are small, e.g., as encountered in breast imaging, $t_{f}\left(\vec{r}, \vec{r}_{0}\right)$ can be approximated $\operatorname{as}^{15}$

$$
t_{f}\left(\vec{r}, \vec{r}_{0}\right)=\int_{L\left(\vec{r}, \vec{r}_{0}\right)} \frac{d^{3} \vec{r}^{\prime}}{c\left(\vec{r}^{\prime}\right)}
$$

where $L\left(\vec{r}, \vec{r}_{0}\right)$ is the line connecting $\vec{r}$ and $\vec{r}_{0}$. It can be shown that

$$
\hat{g}\left(\vec{r}_{0}, \bar{t}\right)=\int d \vec{r} A(\vec{r}) \delta\left[\bar{t}-c t_{f}\left(\vec{r}, \vec{r}_{0}\right)\right] \frac{c t_{f}\left(\vec{r}, \vec{r}_{0}\right)}{\left|\vec{r}_{0}-\vec{r}\right|},
$$

where $\hat{g}\left(\vec{r}_{0}, \bar{t} \equiv c t_{0}\right) \equiv \frac{4 \pi}{\eta} t_{0} \int_{0}^{t_{0}} d t \hat{p}\left(\vec{r}_{0}, t\right)$. Equation $(7)$ is a generalized Radon transform that describes weighted integrals of $A(\vec{r})$ over surfaces $\bar{t}=c t_{f}\left(\vec{r}, \vec{r}_{0}\right)$ that are not spherical in general. It can be shown readily that when $c(\vec{r})=c$, Eqn. (7) reduces to Eqn. (2). If the acoustic speed distribution $c(\vec{r})$ is known, one can attempt to invert Eqn. (7) for accurate reconstruction of $A(\vec{r})$. However, such information is not available in current implementations of TAT.

For many objects of practical interest, the use of a half-time reconstruction method that assumes $c(\vec{r})=c$ can mitigate image artifacts due to acoustic heterogeneities. To understand this heuristically, we can consider the 2D problem and consider the acoustic velocity distribution shown in Fig. 2-(b). This distribution is comprised of two uniform concentric regions that have acoustic velocities $c_{0}$ and $c_{1}$, with $c_{0} \neq c_{1}$, and radii $r_{0}$ and and $r_{1}$, 
respectively. This type of heterogeneous velocity distribution could represent an acoustic model of the female breast, ${ }^{15}$ for example. We will assume that the homogeneous matching medium (that the object is embedded in) also has an acoustic velocity $c_{0}$.

An important observation is that the magnitude of this difference between $g\left(\vec{r}_{0}, \bar{t}\right)$ and $\hat{g}\left(\vec{r}_{0}, \bar{t}\right)$ will be smaller, in general, for small values of $\bar{t}$ than for large values of $\bar{t}$. This is because $\left|t_{f}\left(\vec{r}, \vec{r}_{0}\right)-\frac{\left|\vec{r}_{0}-\vec{r}\right|}{c_{0}}\right|$ will become generally larger as the path-length through the acoustic heterogeneity that the pressure wavefield must propagate increases. This effect is demonstrated in Fig. 2-(b) in which a heterogeneous acoustic speed distribution $c(\vec{r})$ is assumed that is comprised of two uniform disks. Superimposed on $c(\vec{r})$ are solid and dashed lines that represent the curves $\bar{t}=\frac{\left|\vec{r}_{0}-\vec{r}\right|}{c_{0}}$ and $\bar{t}=c_{0} t_{f}\left(\vec{r}, \vec{r}_{0}\right)$ over which the imaging models in Eqns. (2) and (7) integrate over. These curves were calculated analytically assuming the values $c_{1} / c_{0}=1.07, r_{0}=0.92, r_{1}=0.54$, and $R_{0}=1.2$ (arbitrary units), respectively.

These observations indicate that for a large class of objects, when acoustic heterogeneities are present, the complete data function $\hat{g}\left(\vec{r}_{0}, \bar{t}\right)$ will become less spherical Radon transform-like as $\bar{t}$ increases. This results because the temporal components of the photoacoustic wavefields that correspond to large values of $\bar{t}$ are required to propagate through the acoustic heterogeneity over longer optical path-lengths in general and are therefore often more perturbed by the acoustic heterogeneity than are certain components that correspond to smaller values of $\bar{t}$. This indicates that images reconstructed from half-time data functions $g^{h t}\left(\vec{r}_{0}, t\right)$ can contain reduced artifacts levels as compared to images reconstructed from complete data functions. Numerical examples of this are provided in Section 4.

\subsection{Statistical motivation for half-time image reconstruction}

Half-time reconstruction methods can also facilitate statistically optimal image reconstruction. With consideration of data noise, the measured data can be expressed as

$$
\boldsymbol{p}\left(\vec{r}_{0}, t\right)=p\left(\vec{r}_{0}, t\right)+\boldsymbol{n}\left(\vec{r}_{0}, t\right)
$$

where $\boldsymbol{n}\left(\vec{r}_{0}, t\right)$ is an additive noise component that models stochastic variations introduced by the measurement process. Here and elsewhere, boldface letters will denote stochastic quantities. If $\boldsymbol{n}\left(\vec{r}_{0}, t\right)$ is an uncorrelated random variable, the variance of the the data function $\boldsymbol{g}\left(\vec{r}_{0}, \bar{t}\right)$ is given by

$$
\operatorname{Var}\left\{\boldsymbol{g}\left(\vec{r}_{0}, \bar{t}\right)\right\}=\left(\frac{4 \pi \bar{t}}{\eta c}\right)^{2} \int_{0}^{\frac{\bar{t}}{c}} d t^{\prime} \operatorname{Var}\left\{\boldsymbol{p}\left(\vec{r}_{0}, t^{\prime}\right)\right\}
$$

Because $\operatorname{Var}\left\{\boldsymbol{p}\left(\vec{r}_{0}, t^{\prime}\right)\right\}>0$, Eqn. (9) indicates that $\operatorname{Var}\left\{\boldsymbol{g}\left(\vec{r}_{0}, \bar{t}\right)\right\}$ is a monotonically increasing function of $\bar{t}$. This observation suggests that a reconstruction approach that utilizes the half-time data function $g^{h t}\left(\vec{r}_{0} ; \bar{t}\right)$ may be more statistically favorable than one that employs the complete data function $g\left(\vec{r}_{0} ; \bar{t}\right)$ because an estimate of $A(\vec{r})$ can be obtained without use of the measurement data that are corrupted most by noise.

In order to reconstruct an image $A(\vec{r})$ that has an optimally reduced variance without introducing a bias, one can exploit statistically complementary information that is contained in the complete data function $g\left(\vec{r}_{0}, \vec{t}\right)$. Let $\boldsymbol{A}(\vec{r}), \boldsymbol{A}^{h t}(\vec{r})$ denote estimates of $A(\vec{r})$ that are reconstructed from noisy realizations of the complete and half-time data functions $\boldsymbol{g}\left(\vec{r}_{0}, \bar{t}\right)$ and $\boldsymbol{g}^{h t}\left(\vec{r}_{0}, \bar{t}\right)$, respectively. In general, these images will contain statistically complementary information that can be utilized to form an estimate of $A(\vec{r})$ that has a reduced variance. For example, we can form the estimate ${ }^{9}$

$$
\boldsymbol{A}^{\omega}(\vec{r})=\omega(\vec{r}) \boldsymbol{A}(\vec{r})+[1-\omega(\vec{r})] \boldsymbol{A}^{h t}(\vec{r}),
$$

where $\omega(\vec{r})$ is a real-valued combination coefficient. From knowledge of the data noise properties, a combination coefficient $\omega$ can be devised that minimizes the variance of the final estimate $\boldsymbol{A}^{\omega}(\vec{r})$.

\section{NUMERICAL STUDIES AND RESULTS}

We conducted computer-simulation studies to demonstrate some salient features of half-time image reconstruction methods for TAT. 


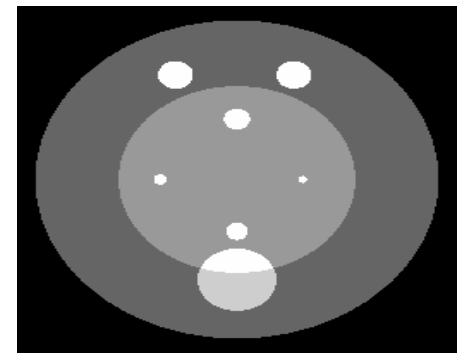

(a)

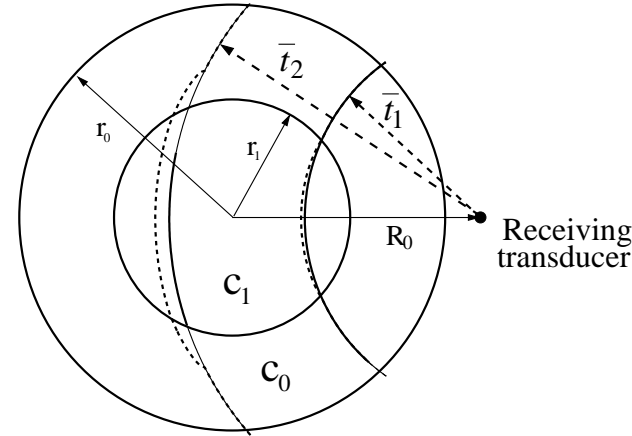

(b)

Figure 2. (a) The numerical phantom for $A(\vec{r})$. (b) The heterogeneous acoustic map $c(\vec{r})$ contains two concentric circles with different speeds of sound denoted by $c_{1}$ and $c_{0}$. Figure (b) also illustrates how the contours that Eqn. (5) integrates (dotted lines) deviate from spherical curves (solid lines) when the object does not have homogeneous acoustic background, here $c_{1}=1.08 c_{0}$.

\subsection{Simulation studies}

We considered the 2D problem, assuming the measurement geometry shown in Fig. 1 with $R_{0}=1.2$ (arbitrary units). The 2D mathematical phantom shown in Fig. 2-(a) was used to represent the electromagnetic absorption map $A(\vec{r})$, which had a circular support of radius $R_{A}=1$. Assuming that the object's acoustic properties were homogeneous and equal to those of the background medium, the complete data function $g\left(\vec{r}_{0}, \bar{t}\right)$ was calculated analytically by use of Eqn. (2). The data function $g\left(\vec{r}_{0}, \bar{t}\right)$ was determined at 360 equally spaced positions $\vec{r}_{0}$ on the scanning aperture and at 256 equally spaced values of $\bar{t}$ over the interval $\left[R_{0}-R_{A}, R_{0}+R_{A}\right]$. We also simulated the case where the object had a heterogeneous acoustic speed distribution $c(\vec{r})$. The acoustic speed distribution $c(\vec{r})$ shown in Fig. 2-(b) was assumed. The acoustic speed values in the two regions satisfied $c_{1}=1.08 c_{0}$, and the radii of the inner and outer disks were $r_{0}=0.92$ and $r_{1}=0.54$. The data function $\hat{g}\left(\vec{r}_{0}, \bar{t}\right)$ was calculated numerically by use of Eqn. (7). The sampling conditions were the same as described above. Noisy versions of the data sets were created by use of the noise model in Eqn. (8), where $\boldsymbol{n}\left(\vec{r}_{0}, t\right)$ was a Gaussian stochastic process with standard deviation $\sigma=0.0075$. Half-time versions of the data sets were created according to Eqn. (4).

Images were reconstructed from the noiseless and noisy complete data sets by use the conventional EM algorithm. ${ }^{14}$ The discontinuity in the half-time data sets introduced by the temporal truncation of the data can result in artifacts in the reconstructed images. To circumvent this effect, a weighted EM algorithm ${ }^{16}$ was employed for reconstruction of images from the noiseless and noisy half-time data sets.

\subsection{Reconstructed images}

Figures 3-(a) and 3-(b) were reconstructed from the noiseless complete and half-time data functions, respectively, corresponding to the acoustically homogeneous object. These two images are virtually identical and accurately represent the true object $A(\vec{r})$. This is consistent with our assertion that accurate images can be reconstructed from half-time data functions in TAT. Figures 4-(a) and 4-(b) were reconstructed from the noisy complete and half-time data functions, respectively. The two noisy images are distinct, with the image reconstructed from the half-time data function having a generally less noisy appearance. In all cases, the EM algorithms were terminated after 120 iterations and the matrix size of the reconstructed images was $256 \times 256$ pixels.

Figure 5 contains reconstructed images of the acoustically heterogeneous object. The images in Figs. 5-(a) and (b) were reconstructed from noiseless and noisy versions of the complete data function, respectively. The images in Figs. 5-(c) and (d) were reconstructed from noiseless and noisy versions of the half-time data function, respectively. In all cases, the EM algorithm was terminated after 120 iterations. It is clear that the images 


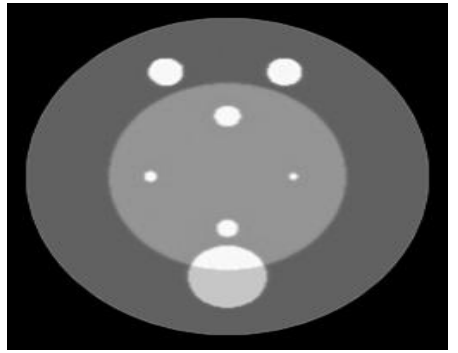

(a)

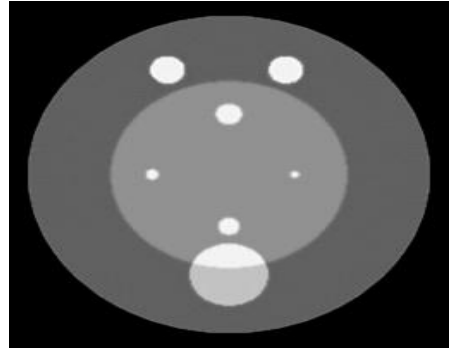

(b)

Figure 3. Images reconstructed from the noiseless (a) full-time and (b) half-time data functions corresponding to the acoustically homogeneous object.

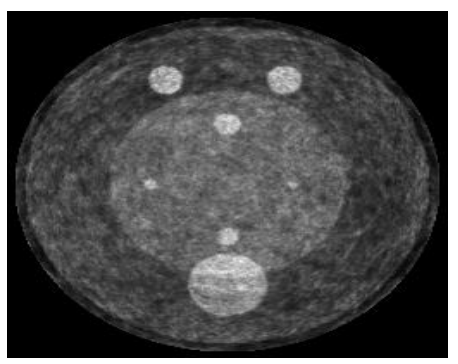

(a)

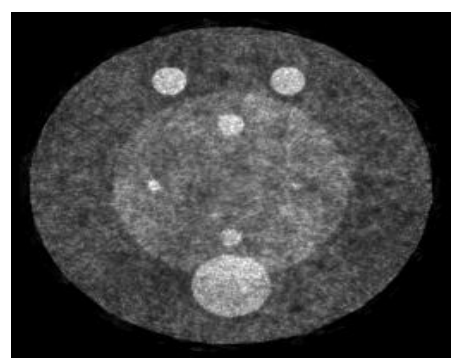

(b)

Figure 4. Images reconstructed from the noisy (a) full-time and (b) half-time data functions corresponding to the acoustically homogeneous object.

reconstructed from the half-time data functions [Figs. 5-(c) and (d)] contain fewer artifacts and lower noise levels than the corresponding images reconstructed from the complete data sets [Figs. 5-(a) and (b)]. This observation corroborates our claim that, in many cases, half-time reconstruction methods for TAT can outperform methods that utilize the complete data function.

\section{SUMMARY}

We have proposed and investigated half-time reconstruction methods for TAT. We demonstrated numerically that the half-time reconstruction methods can mitigate image artifacts due to heterogeneous acoustic properties of an object. Half-time reconstruction methods also facilitate the exploitation of statistically complementary information in the measurement data, which can result in the reduction of image variances. These observations suggest that half-time reconstruction methods may be of significant value for improving image quality in biomedical applications of TAT.

\section{REFERENCES}

1. R. Kruger, W. Kiser, D. Reinecke, G. Kruger, and R. Eisenhart, "Thermoacoustic computed tomography of the breast at $434 \mathrm{MHz}$, IEEE MTT-S Digest 2, pp. 591-594, 1999.

2. R.O. Esenaliev, A. Karabutov, and A. Oraevsky, "Sensitivity of laser opto-acoustic imaging in detection of small deeply embedded tumors," IEEE J. Sel. Top. Quant. 5, pp. 981-988, 1999.

3. A. Beenen, G. Spanner, and R. Niessner, "Photo-acoustic depth-resolved analysis of tissue models," Appl. Spectorsc 51, pp. 51-57, 1997. 


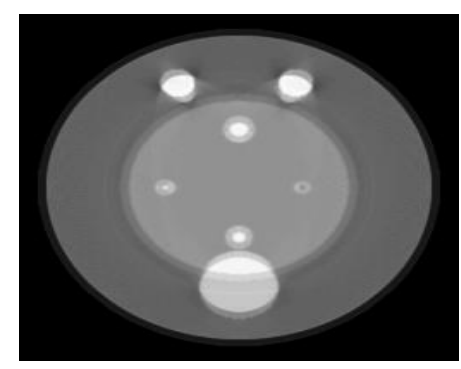

(a)

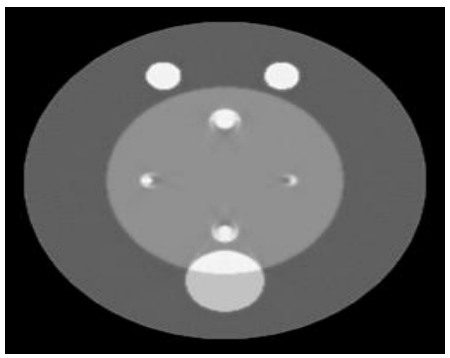

(c)

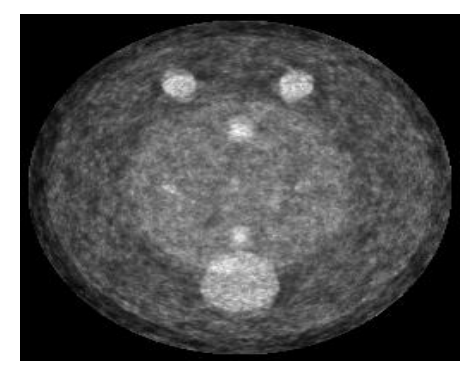

(b)

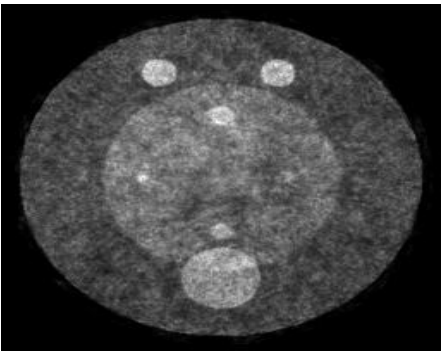

(d)

Figure 5. Top row: images reconstructed from complete data functions corresponding to the acoustically heterogeneous object. Bottom row: images reconstructed from the half-time data functions. Images in the first and second columns were reconstructed from noiseless and noisy versions of the data functions, respectively.

4. M. Xu and L. Wang, "Time-domain reconstruction for thermoacoustic tomography in a spherical geometry," IEEE Transactions on Medical Imaging 21, pp. 814-822, 2002.

5. X. Wang, Y. Pang, G. Ku, X. Xie, G. Stoica, and L.V. Wang, "Non-invasive laser-induced photo-acoustic tomography of the brain in vivo: structural and functional neuroimaging," Nature Biotechnology 21, p. 803, 2003.

6. X. Wang, G. Ku, M. Wegiel, D. Bornhop, G. Stoica, and L.V. Wang, "Noninvasive photoacoustic angiography of animal brains in vivo with near-infrared light and an optical contrast agent," Optics Letters 29:7, pp. 730-733, 2004.

7. M. Xu, Y. Xu, and L.V. Wang, "Time-domain reconstruction algorithms and numerical simulations for thermoacoustic tomography in various geometries," IEEE Transactions on Biomedical Engineering 50, pp. 1086-1099, 2003.

8. M.A. Anastasio, J. Zhang, E. Sidky, Y. Zou, D. Xia, and X. Pan, "Feasibility of half-data image reconstruction in 3D reflectivity tomography with a spherical aperture," IEEE Transactions on Medical Imaging (Submitted), 2005.

9. M.A. Anastasio, J. Zhang, X. Pan, Y. Zou, G. Keng, and L.V. Wang, "Half-time image reconstruction in thermoacoustic tomography," IEEE Transactions on Medical Imaging 24, pp. 199-210, 2005.

10. R. Kruger, P. Liu, R. Fang, and C. Appledorn, "Photoacoustic ultrasound (PAUS) reconstruction tomography," Medical Physics 22, pp. 1605-1609, 1995.

11. S. Norton and M. Linzer, "Ultrasonic reflectivity imaging in three dimensions: Exact inverse scattering solutions for plane, cylindrical, and spherical apertures," IEEE Transactions on Biomedical Engineering 28, pp. 202-220, 1981.

12. D. Finch, S. Patch, and Rakesh, "Determining a function from its mean values over a family of spheres," SIAM Journal of Mathematical Analysis 35, pp. 1213-1240, 2004. 
13. Y. Vardi and D. Lee, "From image deblurring to optimal investments: Maximum likelihood solutions for positive linear inverse problems," Journal of the Royal Statistical Society B 55, pp. 569-612, 1993.

14. X. Pan, Y. Zou, and M.A. Anastasio, "Data redundany and reduced-scan reconstruction in reflectivity tomography," IEEE Transactions on Image Processing 12, pp. 784-795, 2003.

15. Y. Xu and L.V. Wang, "Effects of acoustic heterogeneity in breast thermoacoustic tomography," IEEE Transactions on Ultrasonics, Ferroelectrics, and Frequency Control 50, pp. 1134-1146, 2003.

16. J. Zhang, M.A. Anastasio, X. Pan, and L.V. Wang, "Weighted EM reconstruction algorithms for thermoacoustic tomography," IEEE Transactions on Medical Imaging, (In press), 2004. 